\title{
Gestión Digital Universitaria: Una Propuesta Emergente en Tiempos de Pandemia
}

\section{University Digital Management: An Emerging Proposal in Pandemic Times}

Omar Enrique Figueredo-Díaz \section{(2) EDICIÓN: Resiliencia}

Recibido: 5/julio/2021

Aceptado: 5/octubre/2021

Publicado: 29/noviembre/2021

Páginas: 95-107

\&aís

${ }^{1}$ Venezuela

IIIIInstitución

${ }^{1}$ Universidad José Antonio Páez

\section{Correo Eletrónico} 'ofiguere@gmail.com

\section{(D) ORCID}

${ }^{1}$ https://orcid.org/0000-0003-3924-1111

\section{Citar así: Cf APA / IEEE}

Figueredo-Díaz, O. (2021). Gestión Digital Universitaria: Una Propuesta Emergente en Tiempos de Pandemia. Revista Tecnológica-Educativa Docentes 2.0, 1(1), 95-107.

https://doi.org/10.37843/rted.v1i1.259

O. Figueredo-Díaz, "Gestión Digital Universitaria: Una Propuesta Emergente en Tiempos de Pandemia", RTED, vol. 1, n. ${ }^{\circ}$, pp. 95-107, nov. 2021.

\section{Resumen}

Vida y educación son derechos humanos establecidos en el documento de Declaración de los Derechos Humanos (DDHH) en el cual la Organización Naciones Unidades (ONU), concibe, desde el año 1948. Hasta ese momento, en Venezuela, las Instituciones de Educación Universitaria (IEU) desarrollaban sus funciones de manera presencial, a pesar de que algunas habían iniciado los procesos de aprendizaje mediados por la tecnología. Con base en lo anteriormente expuesto, el Vicerrectorado Académico de la Universidad José Antonio Páez diagnosticó las necesidades administrativas del plan, estableciendo como objetivo diseñar un modelo de gestión digital para garantizar la continuidad de los procesos universitarios, tanto académicos como administrativos. El tipo de investigación fue descriptiva, con un diseño documental, aplicado a una muestra de cuatro (4) facultades y dos (2) direcciones generales. Como resultado se generó una propuesta bajo una visión integradora de cuatro (4) dimensiones: 1) Sistematización de la información, 2) Capacitación del personal, 3) Articulación de los actores, 4) Seguimiento y control del modelo. Las evidencias mostraron que la sistematización de la información y la capacitación del personal en los procesos administrativos son clave de la gestión universitaria, la cual se optimizó al incorporar la tecnología, concluyendo que cuando se fusionan las teorías gerenciales con las herramientas digitales se desarrollan de manera eficiente los procesos propios de la gestión universitaria.

Palabras clave: Gestión Digital Universitaria, propuesta emergente, tiempos de pandemia.

\section{Abstract}

Life and education are human rights established in the Declaration of Human Rights (Human Rights) document in which the United Nations Organization (UN) conceived in 1948. Until then, in Venezuela, the Institutions of University Education (IEU) carried out their functions in person, even though some had started learning processes mediated by technology. As a result, a proposal was generated under an integrating vision of four (4) dimensions: 1) Systematization of the information, 2) Staff training, 3) Articulation of the actors, 4) Monitoring and control of the model. Based on the preceding, the Academic ViceRector of the José Antonio Páez University diagnosed the administrative needs of the plan, establishing the objective of designing a digital management model to guarantee the continuity of university processes, both academic and administrative. The type of research was descriptive, with a documentary design, applied to a sample of four (4) faculties and two (2) general directorates. The evidence showed that the systematization of information and the staff training in administrative processes are crucial to university management, which was optimized by incorporating technology, concluding that when management theories are merged with digital tools, they are developed in an efficient university management process.

Keywords: University digital management, emergent proposal, times of pandemic. 


\section{Introducción}

Vida y educación son derechos humanos establecidos en el documento de Declaración de los Derechos Humanos (DDHH) en el cual la Organización Naciones Unidades (ONU), concibe, desde el año 1948, su artículo 25, que: "Toda persona tiene derecho a un nivel de vida adecuado que le asegure, así como a su familia, la salud y el bienestar..." (1948, p. 36). De igual manera, se consagra con gran relevancia el derecho a la educación al establecer que: "La educación tendrá por objeto el pleno desarrollo de la personalidad humana y el fortalecimiento del respeto a los derechos humanos y a las libertades fundamentales" (1948, p. 36). Igualmente, estos derechos están concebidos como derechos humanos, además de deberes sociales fundamentales, en Venezuela según lo contemplado en su Constitución de la República Bolivariana de Venezuela (CRBV) en sus artículos 83 y 102 .

A los fines de esta investigación se asume como acepción de gestión lo propuesto por Robbins \& Coutler (2005) quienes consideran como gestión el proceso de coordinación de actividades de trabajo, ejecutados de manera eficiente con otras personas y a través de ellas, lo cual se convierte en el objetivo principal de toda gestión. Esta acepción es la que más se aproximaba a los procesos administrativos desarrollados en Instituciones de Educación Universitaria (IEU) venezolanas, de manera síncrona en espacios compartidos por docentes, estudiantes, además de, el personal administrativo, de manera presencial, siendo esta la modalidad bajo la cual se autorizó el funcionamiento de las IEU en Venezuela desde sus inicios por lo cual los procesos administrativos, se gestionaban en los recintos universitarios según los tiempos y espacios definidos para tales fines.

Con la declaración de la pandemia en marzo de 2020 decretada por la Organización Mundial de la Salud (OMS), el Estado venezolano sanciona lo que se conoció como una cuarentena social y colectica en la Gaceta
Oficial $\mathrm{N}^{\circ}$ 6.519, Decreto 4.160, que hizo necesario adoptar "las medidas urgentes, efectivas y necesarias, de protección y preservación de la salud de la población" (2020, p. 1). Adicionalmente el Ministerio del Poder Popular para la Educación Universitaria (MPPEU) venezolano instruyó el Plan Universidad en Casa (Trompiz, 2020). Donde las actividades denominadas "vitales" buscaban garantizar el proceso de prosecución académica de todos los miembros activos en el subsistema de educación universitaria preservando en todo momento las condiciones sanitarias de las comunidades universitarias.

Dentro de este escenario se presentó como alternativa un sistema de educación a distancia mediado por las Tecnologías de la Información y la Comunicación (TIC) con el objetivo de brindar una gestión del conocimiento y el aprendizaje en un entorno seguro. Sin embargo, aun cuando, en Venezuela se había iniciado el proceso de la enseñanza a distancia mediada por las TIC, denominado por Mujica-Sequera (2021) como la alfabetización científica, se observa un escenario en el cual una gran cantidad de IEU comenzaron centrado sus esfuerzos, hasta ese momento, en el proceso de formación del docente, disminuyendo el desarrollo de los procesos administrativos derivados del servicio educativo prestado, por lo cual se llegaron a detener muchos trámites, afectando de manera significativa parte del sistema organizativo propio de una universidad: docentes, estudiantes y personal administrativo.

Estas necesidades llevaron a visualizar alternativas de respuesta entorno al diseño de una propuesta de gestión para garantizar el desarrollo de procesos académicos administrativos a los fines de coadyuvar a garantizar el alcance de los objetivos planteados por el denominado Plan Universidad en Casa. Ante ello se formuló esta interrogante de investigación: ¿Qué elementos deben considerar una propuesta de gestión universitaria digital para garantizar el proceso de prosecución académica de los 
estudiantes en un contexto pandémico? Por ello, se estableció como objetivo principal diseñar una propuesta de gestión digital dirigida a la puesta en funcionamiento del desarrollo de los procesos académicos administrativos propios de una universidad.

\section{Metodología}

El abordaje metodológico para Palella \& Martins (2012), es el conjunto de procedimientos propios en el campo científico para encontrar el valor de verdad. Es una vía o camino para alcanzar una meta o un fin. Con base en ello, para analizar lo requerido por el proceso de gestión administrativa de una universidad en tiempos de pandemia, una vez concluida la fase de concepción del estudio, se procedió a la revisión de la literatura que condujo a tener un marco de análisis para los datos, para ello se aplicó un diseño documental, definido por Arias como un "proceso basado en la búsqueda, recuperación, análisis, critica e interpretación de datos secundarios obtenidos y registrados en fuentes documentales: impresas o electrónicas" (2016, p. 27).

Seguidamente en la fase de diseño, se realizó una investigación de tipo descriptivo, en los términos expuestos por Arias, que la define como "aquella investigación que consiste en la caracterización de un hecho, fenómeno, individuo o grupo con el fin de establecer su estructura o comportamiento" (2016, p. 24). En este caso en particular, se identificaron los requerimientos administrativos de una IEU a los fines de establecer una estructura administrativa ajustada a lo requerido.

Igualmente con un diseño documental, en este caso de estudio se procedió al análisis de todas aquellas normativas emanadas del Ejecutivo Nacional en cuanto al plan de Cuarentena Social, los procesos informados por el MPPEU, los acuerdos del Consejo Universitario como máximo órgano de gobierno de la IEU, unido a los correos electrónicos recibidos por parte de aquellas dependencias que constituyeron el muestreo para el estudio, a partir de los cuales se analizaron las frecuencias de los requerimientos presentados con mayor recurrencia en estas dependencias a los fines de caracterizar lo requerido en cuanto a necesidades administrativas universitarias.

Se realizó un estudio piloto, el cual es definido por Díaz-Muñoz (2020) como breve en factibilidad o viabilidad, para probar aspectos metodológicos de un estudio de mayor escala. En este caso no se consideró el total de las dependencias universitarias. Los resultados del muestreo arrojaron una muestra que estuvo constituida por cuatro (4) facultades: Ingeniería, Ciencias Sociales, Ciencias de la Salud, Facultad de Ciencias Jurídicas y Políticas, unidas a dos (2) grandes direcciones generales: Estudios Básicos y de Estudios de Postgrado.

El tipo de muestreo aplicado fue no probabilístico, de carácter intencional, definido por Arias (2016) como aquel en el cual los miembros de la muestra son elegidos con base en criterios o juicios preestablecidos por el investigador. En este caso, el criterio para seleccionar estas dependencias fue el grado de vinculación directa entre unidad planificadora y las dependencias encargadas de realizar gestión académica, es decir el Vicerrectorado Académico, como unidad planificadora del proceso con las dependencias académicas seleccionas en el muestreo como unidades ejecutoras de lo planificado. El estudio se realizó con una mirada prospectiva, debido a que se esperaba validar a través de una prueba piloto, el desarrollo funcional de la propuesta, con el objetivo de poder expandirlo, posteriormente, en el resto de las dependencias universitarias.

\section{Resultados}

Una universidad se define según el marco legal venezolano como "una comunidad de intereses en la búsqueda de la verdad" (Ley de Universidades, 1970, p. 1). Desde la definición puede considerarse, administrativamente, a la universidad como una organización, es decir, el conjunto de medios con los cuales los miembros de dicha organización trabajan conjuntamente para conseguir metas específicas de manera eficiente (Hernández \& Rodríguez, 2006). 
En este sentido el proceso formación de profesionales, como respuesta a necesidades identificadas en el país y en el mundo, son el principio de agrupación para quienes laboran en una universidad. En el caso venezolano, particularmente en el caso de estudio, una IEU de gestión privada, se presenta un diseño lineal, definido por Gallardo (2011) como una organización con principio de autoridad lineal, donde se presenta una jerarquización de autoridad, definida por la existencia de líneas directas de comunicación entre entes superiores con las dependencias subordinadas.

Las características fundamentales de la organización son:

1. Autoridad única. Representada por el cargo de mayor jerarquía conjuntamente con sus colaboradores, como secuencia de inicio de mando. En este caso, es ejercida por el Consejo Universitario, presidido por el Rector de la mano de sus miembros: el Vicerrector Académico, Secretaria, Decanos de Facultades, los Directores Generales, los Representantes de los Profesores para concluir con los Representes de los Estudiantes.

2. Líneas formales de comunicación. El sistema de comunicación entre dependencias es formal, normalmente siguen líneas del organigrama que representa el sistema organizativo universitario. Para el caso de estudio se considera fundamentalmente: resoluciones del Consejo Universitario, lineamientos de: Rectorado, Vicerrectorado Académico, Secretaría, Decanatos de Facultades como líneas formales de comunicación.

3. Centralización del proceso de toma de decisiones. El origen de toda decisión reside en el Consejo Universitario, a través del Rector, quien informaba los lineamientos ministeriales a los fines de formular los planes estratégicos a seguir para garantizar el desarrollo de actividades académicas administrativas, propiciando con ello el desarrollo de actividades necesarias para los niveles de pregrado, posgrado, además de extensión.

4. Aspecto piramidal. Presenta una visión global de sistema universitario, como organización, es el resultado del incremento del nivel jerárquico en el diseño organizacional del gobierno universitario.

Lo expuesto en el punto anterior se presenta en la Figura 1. De ella se pueden evidenciar sus características como una organización lineal. Por ello era necesario articular el sistema de comunicación entre sus unidades de manera digital, acción que no se había desarrollado hasta el momento pandémico, pues sólo se había desarrollado de manera presencial, síncrona, mediante comunicaciones en físico, denominadas en el argot universitario "oficios", que no son más que comunicaciones escritas con un número de correlativo impresas, firmadas, selladas para su distribución.

Hernández \& Rodríguez (2006), señalan como ventajas de este tipo de organización:

- Presentan una estructura sencilla, de fácil comprensión

- Delimita claramente las responsabilidades de los órganos asociadas a los cargos involucrados.

- Es de fácil implementación

- Presenta una estabilidad considerable

- Es el tipo de organización más indicado para empresas pequeñas. 


\section{Figura 1}

Estructura Organizativa Universitaria

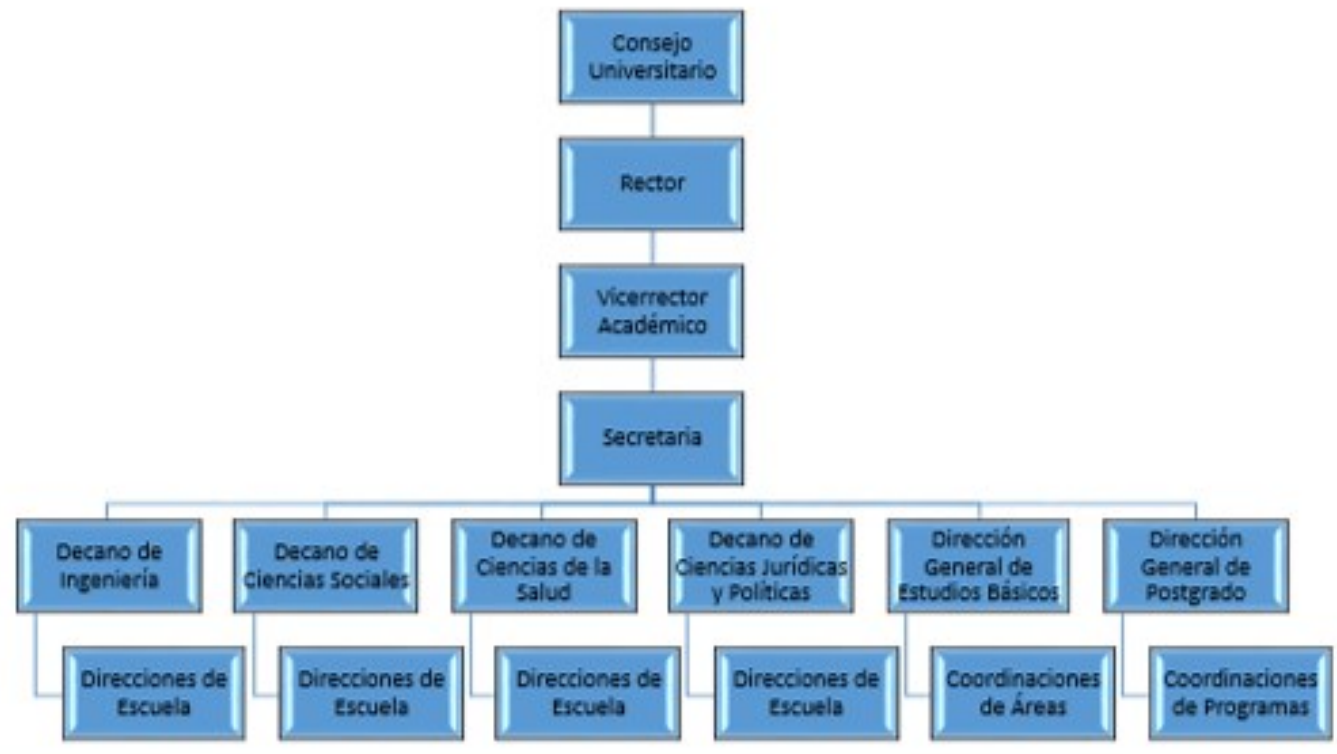

Docentes Docentes Docentes Docentes Docentes Docentes Docentes Docentes Docentes

Nota. La figura muestra las características presentes en las universidades que sumen su estructura desde el modelo de organizaciones lineales, elaboración propia (2021).

Los principios de Gestión de la Propuesta. Las necesidades identificadas por el Vicerrectorado Académico de la IEU caso de estudio, evidenciaban, además del requerimiento de administrar digitalmente a una universidad cuya estructura organizativa era lineal, también demandaba principios que al momento de gestionar digitalmente, pusieran de manifiesto habilidades ya adquiridas por parte del personal en el desarrollo de tareas, debido al poco tiempo disponible para alcanzar una capacitación.

Por ello, se orientó el desarrollo del sistema de gestión con los postulados de Taylor (1856-1915), recogidos en los fundamentos teóricos de la Escuela Científica de Administración. En ellos el enlace selección-entrenamiento adecuado de los operarios, es fundamental para ubicarlos estratégicamente, según sus cualidades en aquellas actividades que les pudieran procurar mejores resultados, junto a un sistema de incentivos
(Melgar \& Leiner, 2008), para así contribuir a un mejor desempeño laboral (Carro \& Caló, 2012).

Con base en estos principios, se formuló una propuesta al Consejo Universitario para modificar los salarios con base en el principio del rendimiento individual: a mayor productividad, mayor cantidad de unidades producidas por el trabajador. Esto, en el contexto universitario se tradujo en una serie de incentivos a otorgar por parte del ente universitario a sus trabajadores, a los fines de que el reconocimiento de la labor realizada influyera en una mejor atención al estudiante, al igual que representaría beneficios para todos los actores del escenario educativo, por cambios repentinos como consecuencia del fenómeno pandémico. A pesar de las críticas de Losada-Sierra (2020) a los postulados de Taylor, son variados los ejemplos donde su implementación se asocia a términos como competencia, de ampliación oportunidades, 
así como de valoración de iniciativa por parte de los empleados (Carro \& Caló, 2012).

\section{La Autoridad Universitaria como Administrador del Proceso de Gestión}

El cargo de autoridad universitaria puede presentar diversas acepciones dentro del propio contexto, sin embargo en este contexto de investigación, se define al Vicerrector Académico, como el administrador eficiente de los recursos humanos e institucionales, con el fin de brindar un servicio educativo de calidad, además de la prosecución académica en tiempos pandémicos haciendo uso de un sistema educativo a distancia mediado por las TIC. Para el logro de tal cometido, debe ejercer cuatro funciones secuenciales del administrador propuestas por Chiavenato (2002), como lo son: Planificación, Organización, Dirección y Seguimiento, funciones para desarrollar con cierto orden lógico con base en los procesos requeridos, como de los objetivos planteados.

Estas teorías se consideran como fundamentos de los principios básicos de los postulados administrativos tradicionales empleados al orientar los procesos de gestión administrativas. Sin embargo, el carácter innovador de lo propuesto subyace en el uso de estas teorías clásicas empleadas por administradores, que han servido de base a los procesos de gestión administrativa universitaria en América Latina (LosadaSierra, 2020), serán implementadas haciendo uso de las herramientas proporcionadas por las TIC, garantizando salud unida a el desarrollo de las actividades académicas de los estudiantes en el subsistema de educación universitaria en tiempos pandémicos.

\section{Información de Base para el Modelo}

La información de base del estudio proveniente de las fuentes señaladas en la Tabla 1, permitió detectar las necesidades, con algunas discrepancias en lo planteado en el abordaje del contexto situacional del contexto investigativo, pero debido al alto índice de frecuencia presentado se consideraron como necesidades reales, sentidas del contexto universitario, razón por la cual se hacía imperativa su atención a los fines de garantizar el desarrollo de los procesos administrativos.

\section{Tabla 1}

Fuentes de Información

Resoluciones del Consejo Universitario

Correos electrónicos de los decanatos y direcciones generales

Redes sociales: Instagram, Twitter

Nota. Fuentes de información consideradas para el diagnóstico de necesidades administrativas, elaboración propia (2021).

Entre estas necesidades de carácter administrativo se encuentran las siguientes:

1. Necesidad de comunicación estudiantes-docentes, docentesescuelas, escuelas-decanatos, decanatos-Vicerrectorado

Académico, Vicerrectorado Académico- Consejo Universitario.

2. Manejo de direcciones de correos electrónicos de manera aleatoria con falta de asertividad en el momento de seleccionar el órgano ante el cual se presentaba el requerimiento.

3. Desconocimiento por parte del estudiantado de las funciones de cada una de dependencias universitarias, evidenciando que se presentaban solicitudes de requerimientos de manera desacertada.

4. Elevadas cantidades de correos electrónicos destinados a dependencias sin competencias para resolver lo planteado.

5. Imposibilidad de comunicación del estudiantado con sus pares académicos, así como, de los docentes de manera grupal.

6. Falta de uniformidad en la información presentada emanada de los Decanatos, Direcciones hacia instancias superiores 
7. Dispersión en los tiempos de entrega de requerimientos presentados.

8. Diversidad no controlada en el uso de herramientas síncronas y asíncronas para el desarrollo de actividades propias del proceso enseñanza-aprendizaje.

9. Diferentes niveles de competencias informacionales de los docentes para el desarrollo de los encuentros académicos, además de, actividades de evaluación.

10. Necesidad de desarrollo de sesiones permanentes del Consejo Universitario bajo modalidad virtual, a los fines establecer los lineamientos o políticas en el contexto pandémico.

11. Difusión de manera deficiente de información, emanada en forma de resoluciones, del Consejo Universitario.

12. Necesidad de estandarización de información fundamental, así como, establecer los tiempos de entrega de acuerdo con necesidades vitales universitarias, tales como, el cumplimiento de actividades académicas por parte de los docentes para el cálculo de pagos por diversos conceptos.

13. Adecuación de normas, requisitos, procedimientos desarrollados de manera presencial, hasta el momento, haciendo uso de las TIC.

Lo anteriormente presentado constituyó los resultados con mayor frecuencia de requerimiento de atención del Vicerrectorado Académico al momento de analizar los documentos recibidos. Posteriormente se contrastó lo postulado en teorías administrativas, lo experimentado por el investigador, unido a los resultados obtenidos al analizar los documentos, a partir de lo cual emergieron cuatro dimensiones que dan forma a lo que se plantea como propuesta de Gestión Digital Universitaria, representado en la Figura 2, de acuerdo con lo descrito a continuación:
Sistematización de la Información.

Definida en el contexto de esta investigación como el proceso mediante el cual se da un orden secuencial a todos los requerimientos identificados. Para lograr comunicar a los distintos niveles que constituyen el sistema universitario, se asumió un diseño organizativo lineal, iniciando por crear formatos en el programa Microsoft Excel, por cada uno de los órganos que requerían de información organizada para lo cual se establecieron las siguientes categorías: nombre de la dependencia, personal responsable, cargo, correo electrónico, además de las actividades en las cuales dicha dependencia era competente.

Como resultado de la compilación de esta información, se creó el Directorio Digital Universitario Docente, figura que se asemeja a lo denominado por Sevilla Sánchez (2021) como comunicación interna en la universidad, en el cual se reflejó información del personal docente en una hoja de Microsoft Excel por cada Dirección de Escuela o Coordinación de Área Curricular: nombre y apellido del docente, asignatura, sección, correo electrónico del docente. De la misma forma, se realizó un proceso de actualización de base datos de los estudiantes, a los fines de conocer en cada listado de clases el número de teléfono celular con los correspondientes correos electrónicos actualizados; de esta manera los docentes pudieron establecer contactos con los estudiantes para acordar estrategias y herramientas para gestión del aprendizaje.

Una vez compilada la información por el Rectorado y el Vicerrectorado Académico procedió a ser analizada, en algunos casos, siguiendo los principios de la Escuela Científica de Administración, reasignando, en algunos casos, al personal según sus habilidades en las tareas a desempeñar. Unido a ello una vez que fueron compilados los formatos diseñados de manera estándar, se publicó la información en redes sociales oficiales universitarias, mediante vínculos e imágenes, estableciendo como punto de inicio el uso de página web como medio principal a partir de la cual se derivaron publicaciones en redes sociales como Twitter 
e Instagram, así como también, se publicaron de manera periódica, los acuerdos del Consejo Universitario, de manera la información comenzara a fluir haciendo uso de los canales virtuales de información establecidos de manera formal.

\section{Figura 2}

\section{Modelo de Gestión Digital Universitaria}

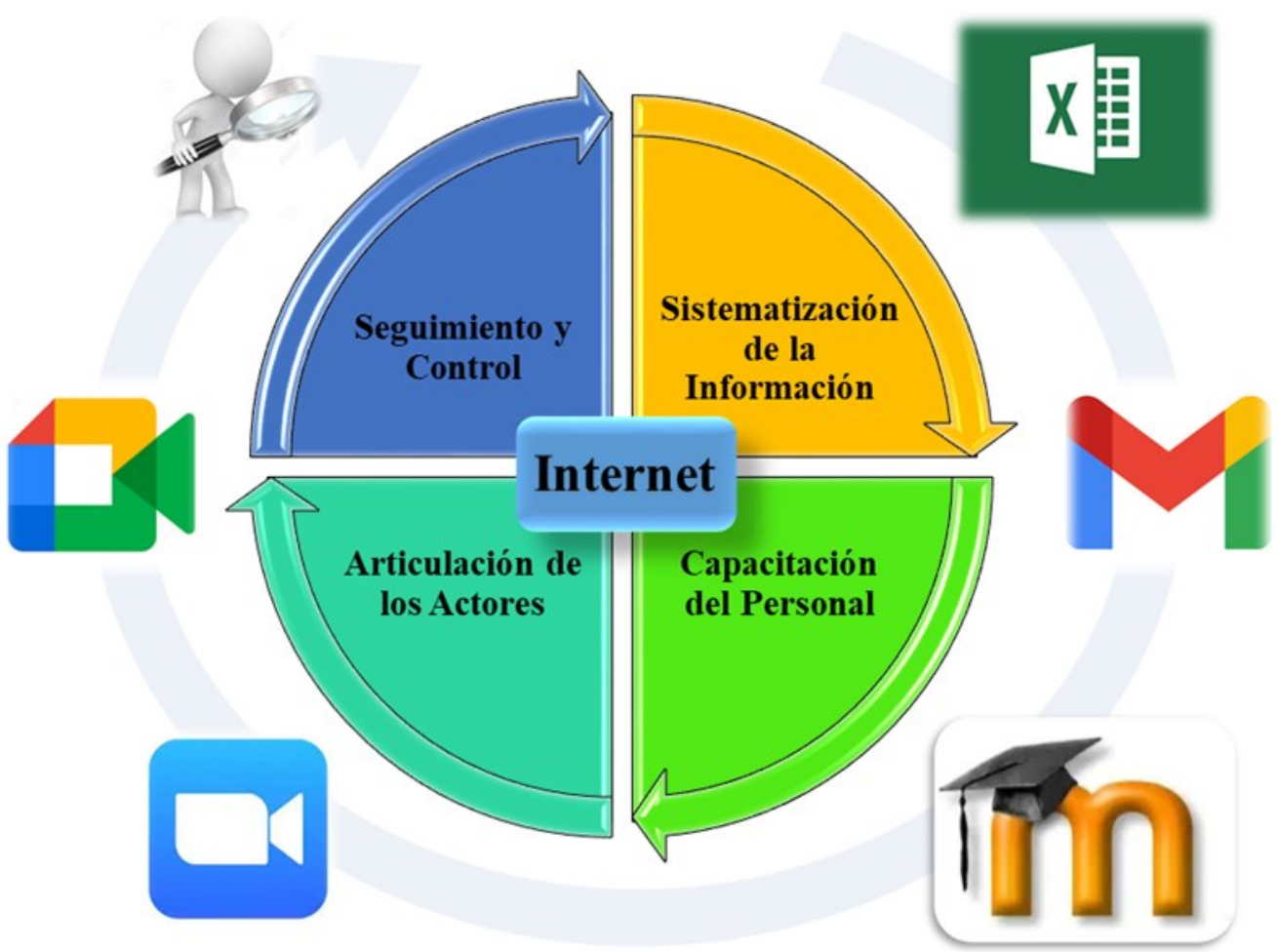

Nota. La imagen representa los procesos de gestión que se cumplen de manera secuencial, así como las herramientas TIC que se fueron incorporando como apoyo al proceso, elaboración propia (2021).

\section{Capacitación del Personal. La IEU} que constituyó el escenario de esta prueba piloto, cuenta con una Dirección General de Nuevas Tecnologías (DGNT) desde el año 2004, Dirección que tiene como misión administrar el Sistema de Gestión de Aprendizaje denominado Acrópolis, el cual está basado en Moodle (por sus siglas en ingles) el cual es código abierto, lo que permite ejecutar servicios en concordancia con los requerimientos de los usuarios.

Hasta el momento en el cual surge el contexto pandémico, Acrópolis había sido empleada como apoyo a prespecialidad, pero debido a sus bondades de interacción con los estudiantes, además del fácil monitoreo para la actividad docente, por lo que fue declarada herramienta prioritaria para gestionar el proceso de aprendizaje por el Consejo Universitario, considerando que esta herramienta se podía combinar con herramientas síncronas como Google Meet o Zoom, para los encuentros explicativos del material presentado en los diseños instruccionales.

Para el mes de marzo del año 2020, solo un $30 \%$ del total de docentes hacían uso de Acrópolis, por esta razón el Vicerrectorado Académico, apoyado en su Dirección General de Nuevas Tecnologías expandió el proceso de formación con el ya existente, en cuanto al diseño de aulas virtuales, bajo la figura del curso de Tutores Virtuales. Con ello se incrementó en tan solo en un periodo lectivo el número de docentes capacitados en el diseño de aulas virtuales a un $83 \%$ del total 
de docentes activos para el periodo lectivo en estudio.

Colateralmente, se incorporaron en el sistema organito universitario los Monitores Virtuales, quienes se definen como un profesional de áreas de conocimientos afines, con formación en el uso de Acrópolis, con el propósito de orientar aquellos pares académicos, expertos de los contenidos, pero carentes de habilidades digitales en cuanto al diseño de aulas virtuales.

De igual manera, en atención a las necesidades del estudiante, la Dirección General de Estudios Básicos y Generales, encargada de recibir a los estudiantes de nuevo ingreso, realizó jornadas de bienvenidas para presentar rutas básicas de acceso tanto al sistema administrativo como al sistema de gestión académica Acrópolis. En este mismo orden de ideas, se desarrolló para los estudiantes regulares una serie de publicaciones periódicas en redes sociales universitarias de las rutas a seguir para acceder a ambos sistemas.

En lo técnico, también se requirieron esfuerzos. El personal adscrito a DGNT actualizó el sistema Moodle lo que requirió un proceso de familiarización con nuevas interfaces, así como, con los nuevos recursos con los cuales se comenzó a contar. En este orden de ideas también, se realizaron inversiones en hospedaje virtual para el alojamiento del sistema, con la finalidad de brindar más estabilidad en la ejecución de esta nueva versión de Acrópolis con todos sus recursos, ello requirió la asesoría de un programador externo para asesorar técnicamente al personal que acometió el proceso de programación del nuevo servidor.

Unido a esta política de capacitación del personal, se estableció, como apoyo a la gestión universitaria, un sistema de reconocimiento al esfuerzo realizado por el trabajador, cumpliendo con ello con otro de los principios clásicos del modelo de Escuela Científica administrativa, relacionado con el sistema de remuneración. Con base en ello, se crea por primera vez en el proceso gerencia universitaria de esta casa de estudios un Bono de Aula Virtual, como un apoyo al docente para soportar los gastos de conectividad, uso de equipos celulares, de computación, entre otros, adicional a otros incentivos como bonos, tales como: bono de transporte. Todas estas medidas facilitaron el proceso de gestión digital universitaria, debido a que se brindaba apoyo a los miembros del personal docente y administrativo para acceder a los recursos necesarios con la finalidad de garantizar la continuidad de los procesos propios de una IEU.

En cuanto a gestiones administrativas, como: formulación de horarios, inscripciones, consignación de formatos de actividades de aprendizaje, planes de evaluación, se diseñaron formatos contentivos de los datos de los estudiantes, asociando las solicitudes planteadas, con columnas por cada nivel de control, los cuales eran compartidos mediante enlaces de drive con todas dependencias administrativas involucradas, encargadas de completar el campo de información correspondiente para finalmente remitir lo recabado a la Dirección de Control de Estudios en donde se procesaban las peticiones aceptadas o rechazadas y los horarios cuya modificaciones fueron procedentes.

Para implantar este proceso el Vicerrectorado Académico conjuntamente con la Coordinación de Auditoría Académica, sostuvieron encuentros síncronos, haciendo uso de Google Meet para desarrollar reuniones de trabajo con los responsables de los procesos, articulando los aportes recibidos en un sistema de trabajo de colaborativo para construir los indicadores e instrumentos, así como, los procesos de inducción de los sistemas administrativo y académico antes mencionados.

En cuanto al cumplimiento del requisito del Servicio Comunitario, se cumplió con lo instruido por el MPPEU en relación con su modalidad de desarrollo, es decir virtual, totalmente a distancia, por lo cual se diseñó un aula virtual contentiva de normativas, reglamentos, formatos de proyectos, empleando los foros electrónicos para consulta de dudas como canal de comunicación. Esta aula fue replicada en todas las facultades, acompañada del uso de Google Meet en jornadas de inducción. El 
abordaje a comunidades en los diversos proyectos se realizó a través de redes sociales, Twitter e Instagram a los fines de desarrollar campañas de concientización de medidas de bioseguridad, así como, orientación al de abordaje de los contextos pandémicos en diversas comunidades.

También se capacitó a comunidades próximas al entorno de los estudiantes en cuanto a liderazgo, motivación personal, formación de emprendedores, todo ello bajo la supervisión de docentes expertos en el área, generando de esta manera el aprovechamiento del tiempo disponible en comunidades receptoras del servicio para procesos de formación y actualización a través el uso de las herramientas tecnológicas con lo cual se resguardó, en todo momento, la salud del estudiante y las comunidades que fueron abordadas de manera digital.

Articulación de los Actores. Los actores del este escenario digital de gestión estuvo constituido por los estudiantes, el personal docente unido a los miembros del personal directivo administrativo universitario, quienes hicieron uso de la internet como elemento articulador entre funciones necesarias para continuar al proceso administrativo. Una vez sistematizados los datos, el medio de comunicación empleado fueron los correos que conformaron el directorio digital institucional, en combinación con herramientas síncronas como Zoom o Google Meet asociadas a los correos electrónicos creados para fines institucionales, por lo cual se reconocía muy fácilmente a la dependencia contactada, así como, el trabajador responsable de la respuesta emitida.

Este uso de la internet como articulador en el modelo de gestión es recursivo, pues se manifiesta como herramienta de comunicación del estudiante con el profesorado, además de la universidad. De igual manera, conectó a la universidad, con el profesorado y a la población estudiantil, así como, también conecto al docente con su universidad y grupos de estudiantes, todo ello unido al uso de redes sociales o correos electrónicos sustentados en el servicio de interconexión que brinda la internet como recurso fundamental.

Seguimiento y Control. Como todo sistema de gestión, el seguimiento de lo planificado para alcanzar los objetivos organizacionales es necesario, incluso esta es una actividad continua del administrador. Desde una visión de gestión universitaria, la función de seguimiento emergió desde el requerimiento de observar el cumplimiento de las actividades a desarrollar por parte de los estudiantes, del profesorado, así como, del personal directivo administrativo que presta servicios en este contexto universitario.

En medio del proceso pandémico, también se fortaleció la Coordinación de Auditoría Académica, apoyada en los monitores virtuales quienes bridan acompañamiento al docente, unido a los reportes de actividades virtuales, presentados de acuerdo a un cronograma de fechas establecidas, ante los respectivos Coordinadores de Áreas Curriculares, Directores de Escuelas, Decanos, Directores Generales, para finalmente compilar todos los reportes en la Coordinación de Auditoría Académica, adscrita al Vicerrectorado Académico.

El propósito de esta unidad de auditoría en el contexto académico no es de carácter punitivo, por el contrario, su misión es brindar apoyo a los miembros del personal docente para alcanzar los niveles deseados en el proceso de gestión del aprendizaje con su correspondiente evaluación, estableciendo para el caso particular de esta comunidad universitaria las siguientes actividades administrativas derivadas del acto docente considerados como indicadores de desempeño:

1. Consignación de planes para actividades de aprendizaje o evaluación por cada unidad temática a desarrollar.

2. Análisis del informe de monitoreo virtual con base a los indicadores de interacción del diseño instruccional establecido empleando una rúbrica de valoración previamente establecida 
3. Análisis del cumplimiento de actividades docentes informado por los miembros del personal Directivo (Decanos y Directores Generales)

4. Análisis del cumplimiento de actividades administrativas del docente, tales como: carga de notas, cierre de actas en el periodo lectivo correspondiente entre otras.

El modelo de gestión propuesto presenta cuatro dimensiones interconectadas gracias a la internet, espacio virtual donde se realiza una combinación de paquetes, plataformas, programas a través de los cuales se facilita el proceso de interacción entre los actores de cada dimensión alcanzando, de esta manera, el dinamismo requerido para el desarrollo de actividades administrativas propias de una IEU en un contexto de educación a distancia mediada por el uso de los recursos tecnológicos.

En lo concerniente al personal netamente administrativo, el Rectorado de esta IEU, estableció un formato para el informe de actividades administrativas, conjuntamente con un cronograma para su consignación, previendo fechas antes del proceso de elaboración de nóminas para el pago, con el fin de presentar oportunamente la información ante la Dirección de Recursos Humanos, los resultados del trabajo administrativo, realizado a distancia, haciendo uso del internet, para su correspondiente remuneración unido al pago de incentivos.

\section{Conclusiones}

Una vez revisados los resultados del estudio, el proceso de investigación se orienta a concluir sobre la factibilidad favorable para sistematizar los procesos administrativos, originando un sistema de gestión, el cual perfectamente puede ser desarrollado a través de herramientas telemáticas, requiriendo siempre del gerente, los operadores quienes unidos a los usuarios imprimen el toque humano a cada uno de los procesos gestionados detrás el computador. Por lo tanto, el éxito de un sistema de gestión digital universitario depende del compromiso con el cual cada uno de los miembros del equipo asuma como suya las tareas digitales asignadas, desarrollando con ello el sentido de pertenencia con su institución a través del desarrollo del trabajo colaborativo. Otras reflexiones que surgen del estudio son las que se exponen a continuación:

Los sistemas de gestión sustentados en teorías administrativas tradicionales pueden ejecutarse de manera digital haciendo uso de herramientas, plataformas $\mathrm{o}$ paquetes digitales disponibles a través del internet como herramienta.

Para abordar cualquier sistema de gestión, es necesario conocer los rasgos característicos organizacionales, a los fines de identificar los principios teóricos administrativos, definidos por Sánchez, Ríos, Cajas \& Tanqueño (2021) como conducta organizacional, a través de los cuales se puedan sistematizar los procesos gerenciales.

Una información ordenada es el punto de partida para diseñar procesos digitales de gerencia universitaria. Concluyendo, al igual que Sevilla-Sánchez (2021), en lo relacionado a enfatizar el uso del correo institucional y hacer más llamativa la información acordada.

Una universidad, desde el punto de vista administrativo, es una organización orientada con fundamentos gerenciales adaptados a los desarrollos tecnológicos propios del contexto social del conocimiento.

Los procesos administrativos de atención al estudiante, tales como inscripciones, solicitudes especiales, presentaciones de trabajos de grado se pueden realizar completamente a distancia, partiendo de un proceso planificado de manera secuencial haciendo uso del internet como herramienta.

El cumplimiento del Servicio Comunitario como requisito para obtener el título profesional, en el caso venezolano, se puede desarrollar a través de redes sociales conjuntamente con plataformas síncronas o asíncronas. Sin embargo, se debe considerar el contexto de algunos sectores en los cuales 
no se cuenta con acceso a internet, ni los dispositivos de conexión necesarios.

El personal docente, así como, el personal administrativo debe ser capacitado en el uso de herramientas digitales, a los fines de desarrollar lo que Rodríguez, Fueyo \& Hevia (2021) denominan competencias digitales para experiencias educativas innovadoras, con la finalidad de desarrollar actividades con las cuales se contribuya al proceso de gestión digital universitaria con asertividad.

El proceso gerencial de una IEU debe ser administrado por sus autoridades, por lo cual deben mantener presente, de manera constante, las actividades fundamentales del administrador como lo son: Planificación, Organización, Dirección y Seguimiento. Estas actividades deben revisarse constantemente de manera a los fines de actualizar los planes de gestión con el objeto de brindar una mejor atención a los miembros de comunidades universitarias, garantizando con ello el acceso a un sistema educativo de calidad.

Un sistema de gestión digital colabora con el Estado en preservar los Derechos a Salud y Educación mediante el uso de herramientas digitales que brinda el internet.

En un contexto pandémico, el desarrollo e implementación de un sistema de gestión digital universitario garantiza el acceso al sistema educativo, formado profesionales de un contexto de bioseguridad.

Finalmente, este proceso de investigación también genera reflexiones de carácter prospectivo, sustentados en los aspectos experimentados durante el proceso de implantación de esta propuesta emergente durante el desarrollo del proceso de cuarentena social experimentado en Venezuela y el mundo, considerando necesario validar la propuesta en el resto de las dependencias académico administrativo que conforman la totalidad del contexto de estudio.

Con base en lo anteriormente expuesto el autor, considera importante compartir este estudio con diversas comunidades de investigadores a nivel mundial con el objetivo de someterla a valoración en otros escenarios, así como, la realización de aproximaciones sucesivas al sistema de gestión implantado, haciendo uso del método de educación comparada, en los diversos contextos en los cuales se pueda requerir un sistema de gestión universitaria digital.

Aspectos como la evaluación de la infraestructura, tanto física como tecnológica, son temas pendientes en el ámbito de la gestión universitaria. Igualmente, se considera necesario realizar contrastes con otras propuestas de gestión a fin de aproximar buenas prácticas, así como indicadores de calidad para la gestión de digital universitaria.

\section{Reconocimiento}

H.G.P. reconocimiento del autor por el apoyo en cada momento de este proceso de gestión. M.A.F.P. reconocimiento del autor por apoyo en el proceso de compilación de información. A todo el equipo del V.R.A.C. sin quienes no existiría alguna propuesta de gestión.

\section{Referencias}

Arias, F. (2016). El Proyecto de Investigación. Editorial Episteme.

Carro, F. D., \& Caló, A. (2012). La Administración Científica de Frederick W. Taylor: una lectura contextualizada. VII Jornadas de Sociología de la UNLP. Bahía Blanca,

RepúblicaArgentina.https://repositoriodigital.uns.ed u.ar/bitstream/handle/123456789/4435/Administraci $\% \mathrm{c} 3 \% \mathrm{~b} 3 \mathrm{n} \% 20$ cient $\% \mathrm{c} 3 \%$ adfica.pdf? sequence $=3 \&$ is Allowed $=\mathrm{y}$

Chiavenato, I. (2002). Gestión del Talento Humano. Mc Graw Hill Interamericana.

Constitución Nacional de la República Bolivariana de Venezuela. (1999). Gaceta Oficial $N^{\circ}$ 5908. 17-30 http://www.minci.gob.ve/wpcontent/uploads/2011/04/CONSTITUCION.pdf

Decreto $\mathrm{N}^{\circ}$ 4. 160. (2020). Estado de Alarma para atender la Emergencia Sanitaria del Coronavirus (COVID-19). Gaceta Oficial $\mathrm{N}^{\circ} \quad 6.519 \quad$ Extraordinario. http://extwprlegs1.fao.org/docs/pdf/ven194367.pdf

Declaración Universal de Derechos Humanos. (1948). $\begin{array}{llll}\text { Resolución } & 217 \quad A \quad \text { (III). } & \text { 34-37. }\end{array}$ https://undocs.org/es/A/RES/217(III) 
Díaz-Muñoz, G. (2020). Metodología del estudio piloto. Revista chilena de radiología, 100-104. http://dx.doi.org/10.4067/S071793082020000300100

Gallardo, E. (2011). Fundamentos de Administración. Universidad de Barcelona http://diposit.ub.edu/dspace/bitstream/2445/17604/6 /Fundamentos\%20Administracion\%20EGallardo.pd $\mathrm{f}$

Hernández, S. \& Rodríguez, S. (2006). Introducción a la Administración. McGraw Hill Interamericana.

Ley de Universidades. Gaceta Oficial No. 1429 Extraordinario.8 de septiembre de 1970. http://www.ucv.ve/fileadmin/user_upload/comision _electoral/Normativa_legal/index.-.htm\#: :text=Ley \%20de\%20Universidades\%20-

Losada-Sierra, M. (2020). La administración científica de la educación y la condición contingente del ser humano. Educación y Sociedad. No. 41, 1-13. doi: https://doi.org/10.1590/ES.227542

Melgar-Bayardo, J., \& Leiner-De la Cabada, M. (2008). La efectividad de los sistemas de incentivos y su relación con el desempeño laboral. Global Conference on Business and Finance.425428.https://www.researchgate.net/profile/JangLee/publication/228425229_Related_and_Unrelated Corporate_Diversification_and_Firm_Value_Evide nce_from_Korean_Business_Groups/links/563b153 908aeed $0 \overline{5} 31 \mathrm{dccc} \overline{6} \mathrm{a} /$ Related-and-UnrelatedCorporate-Diversification-and-F

Mujica-Sequera, R. (2021). Alfabetización Científica: Herramienta Indispensable en la Era Digital. Revista Internacional Tecnológica - Docentes 2.0. Vol. 11. Núm. $1.19-26$. https://doi.org/10.37843/rted.v11i1.181

Organización Panamericana de la Salud (OPS) (2020). La OMS caracteriza a COVID-19 como una pandemia. https://www.paho.org/es/noticias/11-3-2020-omscaracteriza-covid-19-como-pandemia

Palella, S., \& Martins, F. (2012). Metodología de la investigación cuantitativa. Fondo Editorial de la Universidad Pedagógica Experimental Libertador.

Robbins, S. \& Coulter, M. (2005). Administración. 8va edición. Pearson Educación.

Rodríguez, C., Fueyo, A. \& Hevia, I. (2021). Competencias digitales del profesorado para innovar en la docencia universitaria Pixel-Bit. Revista de Medios y Educación. Editorial Universidad de Sevilla. 61 (7198).

https://recyt.fecyt.es/index.php/pixel/article/view/86 $305 / 64872$

Sánchez, I., Ríos, M., Cajas, V. \& Tanqueño, O. (2021). Liderazgo Positivo en Organizaciones. Revista Venezolana de Gerencia. 26 (25). 544-563. https://www.produccioncientificaluz.org/index.php/r vg/article/view/36266/38791
Sevilla-Sánchez, M. (2021). Manejo de la Comunicación Interna en la Universidad de los Hemisferios. chrome-

extension://efaidnbmnnnibpcajpcglclefindmkaj/view er.html?pdfurl=http $\% 3 \mathrm{~A} \% 2 \mathrm{~F} \% 2 \mathrm{Fdspace}$. uhemisferi os.edu.ec\%3A $8080 \% 2$ Fxmlui $\% 2$ Fbitstream $\% 2$ Fhan dle $\% 2$ F $123456789 \% 2$ F $1248 \% 2$ FTF\% $\% 2520$ MARIA \%2520EMILIA\%2520SEVILLA\%2520SANCHEZconvertido.pdf $\% 3$ Fsequence $\% 3 \mathrm{D} 1 \% 2$ is Allowed $\%$ 3 Dy\&clen $=804151$

Trompiz, C. (2020). Plan Universidad en Casa. $\mathrm{http}: / /$ universidadencasa.educacionuniversitaria.gob .ve/nextcloud/index.php/s/Dd2jcnjZiN8d9mT\#pdfv iewer 